\title{
Fisioterapia aquática aplicada em recém-nascidos e crianças: uma revisão integrativa
}

\author{
Aquatic physical therapy applied in newborns and children: a comprehensive review \\ Fisioterapia acuática aplicada en recién nacidos y niños: una revisión integrativa
}

Daniela Cristina Rambo ${ }^{1 *}$, Amanda Quadros de Souza ${ }^{2}$, Cristina Saling Kruel ${ }^{3}$, Nadiesca Taisa Filippin $^{3}$.

\section{RESUMO}

Objetivo: Realizar uma revisão integrativa acerca da aplicação da fisioterapia aquática em recém-nascidos ou crianças, conhecida também como "hidroterapia". Metodologia: A busca de referências foi realizada nas bases de dados PubMed, SciELO, Biblioteca Virtual em Saúde (BVS) utilizando os seguintes descritores de saúde: "hydrotherapy", "infants", "newborn", e no Google Acadêmico as palavras-chaves: "hidroterapia", "recém-nascidos", "crianças" e "fisioterapia aquática" durante o período de 2007 a 2017. Resultados: Foram encontrados 8 estudos que preencheram os critérios de inclusão e exclusão desse trabalho. Os resultados demonstraram que a fisioterapia aquática constitui um recurso seguro para sua aplicação em recém-nascidos e crianças em diversas temáticas como: dor, desenvolvimento neuromotor e bem-estar. Considerações Finais: $O$ que se conclui dos estudos analisados, podemos considerar que existe escassez na literatura quanto ao tema fisioterapia aquática em recém-nascidos ou crianças. São necessários mais estudos clínicos bem delineados para análise da aplicação da fisioterapia aquática para este público e seus benefícios.

Palavras-chave: Hidroterapia, Fisioterapia aquática, Crianças, Recém-nascidos, Benefícios.

\begin{abstract}
Objective: To carry out an integrative review on the application of physiotherapy in newborns or children, also known as "hydrotherapy". Methodology: The search for references was performed in PubMed, SciELO, Virtual Health Library (VHL) databases using the following health descriptors: "hydrotherapy", "infants", "newborn", and in Google Scholar the keywords: "Hydrotherapy", "newborn", "children" and "aquatic physiotherapy" during the period 2007 to 2017. Results: We found 8 studies that met the inclusion and exclusion criteria of this study. Final considerations: Given the analysis of the studies we can consider that there is a shortage in the literature on the subject of aquatic therapy in newborns or children. More welldesigned clinical studies are needed to analyze the application of aquatic physiotherapy to this public and its benefits.
\end{abstract}

Keywords: Hydrotherapy, Aquatic physiotherapy, Children, Newborns, Benefits.

\section{RESUMEN}

Objetivo: Realizar una revisión integrativa sobre la aplicación de la fisioterapia en recién nacidos o niños, también conocida como "hidroterapia". Metodología: La búsqueda por referencias fue realizada en las bases de datos PubMed, SciELO, Biblioteca Virtual en Salud (BVS) utilizando los siguientes descriptores de salud: "hidroterapia", "infants", "newborn", y en Google Académico las palabras clave: "Hidroterapia", "recién nacidos", "niños" y "fisioterapia acuática" durante el período de 2007 hasta 2017. Resultados: Se encontraron 8 estudios que cumplieron los criterios de inclusión y exclusión de ese trabajo. Consideraciones Finales: Ante el análisis de los estudios podemos considerar que hay escasez en la literatura en cuanto al tema de fisioterapia acuática en recién nacidos o niños. Se necesitan más estudios clínicos bien delineados para analizar la aplicación de la fisioterapia acuática para este público y sus beneficios.

Palabras clave: Hidroterapia; fisioterapia acuática; niños; recién nacidos; beneficios.

\footnotetext{
${ }^{1}$ Universidade Franciscana (UFN), ljuí- RS, *E-mail: danielarambo@hotmail.com

2Universidade Franciscana (UFN), Cachoeira do Sul- RS

3Universidade Franciscana, Santa Maria-RS.
} 


\section{INTRODUÇÃO}

Entende-se o termo fisioterapia aquática como tratamento pela água, uso tópico ou externo para fins terapêuticos, os benefícios que fornecem a sua aplicação são produzidos pelo conjunto de suas propriedades mecânicas, térmicas e químicas, junto a outros agentes como o movimento (PASCUAL CT, 2014).

A fisioterapia aquática tem sido usada há muitos anos, como uma terapêutica para promover relaxamento, melhora da circulação sanguínea, restaurar mobilidade, melhorar a coordenação motora e promover recreação (BARBOSA LPC, 2012). Embora ainda a literatura é escassa para o uso em recém-nascidos e crianças.

Fisiologicamente, a pressão hidrostática da imersão de água flui o movimento do espaço extra vascular para o intravascular, espaço com potencial para reduzir o sangue, pressão e edema (BRICKHOUSE B, et al., 2016). O exercício físico na água tem proporcionado benefícios físicos em diferentes populações (CARVALHO DR, et al., 2015).

Nos recém-nascidos (RN) os efeitos terapêuticos em imersão na água aquecida proporcionam melhora na circulação sanguínea, na musculatura respiratória aumentando a sua capacidade vital e assim também faz com que o RN se reorganize com os movimentos de sua vida fetal (VIGNOCHI C, et al., 2010).

Em um estudo realizado por Barbosa LCP, et al. (2015) no qual avaliaram o impacto da hidroterapia em recém-nascidos hospitalizados foi encontrada melhora na oxigenação periférica e redução da dor. Valizadeh L, et al. (2017) compararam os efeitos da hidroterapia com exercícios passivos no desempenho motor e desenvolvimento neuromuscular em crianças nascidas prematuras e significativamente os membros inferiores tiveram melhor reação no grupo da hidroterapia. Da mesma forma que Tobinaga WCO, et al. (2016) através da hidroterapia em recém-nascidos demonstraram redução na frequência respiratória $(F R)$ e frequência cardíaca (FC), sugerindo que estas alterações estão associadas a mudança para um estado comportamental de conforto e relaxamento ao recém-nascido, proporcionado pelas propriedades físicas da água.

A fisioterapia aquática é um método não farmacológico que serve para diminuir os desconfortos como a dor e consequentemente melhora da qualidade do sono profundo e pode contribuir para a redução dos efeitos nocivos da Unidade de Terapia Intensiva Neonatal em lactentes, sem privá-los da estimulação tátil-cinestésica necessária para o seu desenvolvimento neurológico (VALIZADEH L, et al., 2017).

Considerando a importância do tema para a saúde infantil o objetivo desse estudo foi realizar uma revisão integrativa sobre a aplicação da fisioterapia aquática em recém-nascidos e crianças.

\section{METODOLOGIA}

Para a elaboração desta revisão integrativa, formou-se a seguinte questão de pesquisa: qual o conhecimento científico produzido na literatura nacional e internacional sobre a aplicação da fisioterapia aquática em recém-nascidos e crianças? Como plano de execução, foram seguidas as seguintes etapas: definição do tema e elaboração da questão de pesquisa; definição dos critérios de elegibilidade para inclusão e exclusão dos estudos; levantamento das publicações nas bases de dados; categorização e análise das informações encontradas nas publicações; avaliação dos estudos selecionados; apresentação dos resultados, incluindo análise crítica dos achados e síntese da revisão.

As buscas dos artigos científicos referentes à questão de pesquisa foram feitas nas bases de dados PubMed, usando o cruzamento dos seguintes descritores do MeSH: "hidrotherapy", "newborn", "infant", SciELO, Biblioteca Virtual em Saúde (BVS- BIREME) usando cruzamentos dos seguintes descritores do DeCS: "hidroterapia", "recém-nascidos", "crianças" no modo de "pesquisa avançada", e no Google Acadêmico foram utilizados as palavras-chaves: "hidroterapia infantil", "fisioterapia aquática", "banho de imersão". Foi realizada a leitura do título de todos os artigos encontrados para saber se havia alguma relação com a questão de pesquisa. A coleta dos artigos foi no mês de junho de 2017. 
Após foi realizada a leitura dos resumos dos artigos, previamente selecionados na primeira etapa e avaliados segundo os critérios de inclusão e exclusão. Os critérios de inclusão foram: artigos que contemplassem o tema proposto, fisioterapia aquática aplicada em recém-nascidos ou crianças, delimitados no período de 2007 a 2017, na literatura nacional e internacional e que haviam sido publicados na íntegra. Os critérios de exclusão estabelecidos foram: artigos que estivessem relacionados com adultos ou idosos, não estivessem disponíveis em português, inglês ou espanhol. Os artigos de interesse foram adquiridos na íntegra e os dados foram coletados para constituir o estudo.

\section{RESULTADOS}

A busca resultou em 44 publicações na base de dados PubMed, 3 publicações na base de dados SciELO, 20 publicações na Biblioteca Virtual em Saúde e 327 publicações no Google Acadêmico. A amostra foi composta por 8 artigos conforme o fluxograma das estratégias de busca na base de dados (Figura 1).

Figura 1 - Fluxograma coleta e análise dos dados.

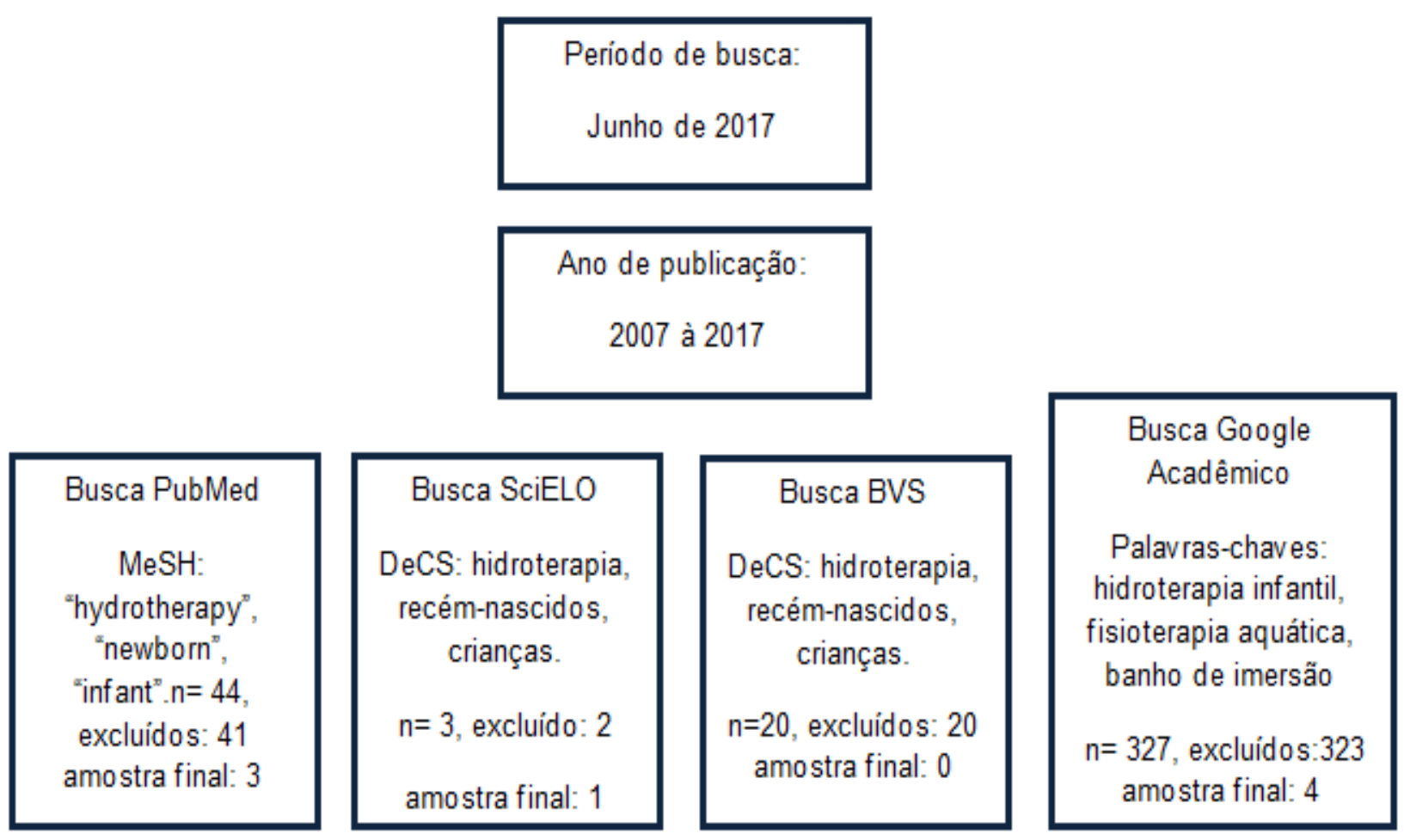

Amostra final:

8 artigos

Fonte: Elaborado pelas autoras, 2019.

Conforme a amostra final foi elaborado um quadro (Quadro 2) que apresenta uma síntese dos principais dados extraídos dos estudos. Relacionado aos autores, ano de publicação, título, base de dados, periódicos, objetivo, método, resultados e conclusão. 


\begin{tabular}{|c|c|c|c|c|c|}
\hline Autores/Ano & Título do Artigo & $\begin{array}{l}\text { Base de dados/ } \\
\text { Periódico }\end{array}$ & Objetivo & Métodos & Principais resultados/Conclusão \\
\hline $\begin{array}{l}\text { SILVA JO et } \\
\text { al., } 2009 .\end{array}$ & $\begin{array}{l}\text { Influência da estimulação } \\
\text { aquática } \\
\text { desenvolvimento no } \\
\text { crianças de } 0 \text { a } 18 \text { meses: } \\
\text { um estudo piloto }\end{array}$ & $\begin{array}{l}\text { SciELO/ } \\
\text { Revista Fisioterapia e } \\
\text { Pesquisa }\end{array}$ & $\begin{array}{l}\text { Verificar a influência } \\
\text { de um programa e } \\
\text { estimulação aquática } \\
\text { no desenvolvimento } \\
\text { de crianças de } 0 \text { a } 18 \\
\text { meses, com ênfase } \\
\text { no desenvolvimento } \\
\text { motor. }\end{array}$ & $\begin{array}{l}\text { Estudo experimental piloto - } 12 \\
\text { crianças. } \\
\text { O desenvolvimento foi avaliado por } \\
\text { meio do teste Denver II e o AIMS. } \\
\text { Para avaliar desenvolvimento } \\
\text { pessoal social, motor fino, motor } \\
\text { grosso e linguagem. A intervenção } \\
\text { da fisioterapia aquática foi realizada } \\
1 \text { vez por semana durante } 8 \\
\text { semanas, } 50 \text { minutos }\end{array}$ & $\begin{array}{l}\mathrm{Na} \text { análise estatística não foram } \\
\text { encontradas diferenças significativas } \\
\text { entre os valores antes e após o } \\
\text { programa de estimulação aquática, } \\
\text { assim, não se pode afirmar que o } \\
\text { estímulo aquático favoreça o } \\
\text { desenvolvimento infantil. }\end{array}$ \\
\hline $\begin{array}{l}\text { VIGNOCHI C, } \\
\text { TEIXEIRA PP, } \\
\text { NADER SS., } \\
2010 .\end{array}$ & $\begin{array}{l}\text { Efeitos da fisioterapia } \\
\text { aquática na dor e no estado } \\
\text { de sono e vigília de recém- } \\
\text { nascidos pré-termo estáveis } \\
\text { internados em unidade de } \\
\text { terapia intensiva neonatal }\end{array}$ & $\begin{array}{l}\text { SciELO } \\
\text { BVS } \\
\text { PubMed/ Revista } \\
\text { Brasileira de Fisioterapia }\end{array}$ & $\begin{array}{l}\text { Avaliar os efeitos da } \\
\text { fisioterapia aquática } \\
\text { na dor e no ciclo } \\
\text { sono e vigília de } \\
\text { bebês prematuros } \\
\text { estáveis } \\
\text { hospitalizados. }\end{array}$ & $\begin{array}{l}\text { Ensaio clínico não controlado de } \\
\text { séries temporais. } \\
\text { Avaliação das variáveis: FC, FR, } \\
\text { PAM, SpO2, temperatura corporal, } \\
\text { dor e comportamento. } \\
\text { Foi realizado } 10 \text { minutos de } \\
\text { fisioterapia aquática, somente uma } \\
\text { intervenção. }\end{array}$ & $\begin{array}{l}\text { Após a realização da fisioterapia } \\
\text { aquática, os recém-nascidos passaram } \\
\text { para um estado de sono profundo. } \\
\mathrm{Na} \text { avaliação da dor NFCS houve } \\
\text { redução significativa do escore. Os } \\
\text { resultados do presente estudo indicam } \\
\text { a melhorados parâmetros fisiológicos, } \\
\text { dos sinais de dor e da qualidade do } \\
\text { sono após a intervenção e sugerem que } \\
\text { a técnica pode ser benéfica aos recém- } \\
\text { nascidos pré-termo }\end{array}$ \\
\hline $\begin{array}{l}\text { MELO FR, } \\
\text { ALVES DAG, } \\
\text { LEITE JMS., } \\
2012 .\end{array}$ & $\begin{array}{l}\text { Benefícios da hidroterapia } \\
\text { para espasticidade em uma } \\
\text { criança com hidrocefalia }\end{array}$ & $\begin{array}{l}\text { Google Acadêmico/ } \\
\text { Revista de Neurociência }\end{array}$ & $\begin{array}{l}\text { Avaliar o efeito da } \\
\text { hidroterapia em uma } \\
\text { criança com } \\
\text { diagnóstico clínico } \\
\text { de hidrocefalia e } \\
\text { diagnóstico } \\
\text { fisioterápico de } \\
\text { quadriplegia } \\
\text { espástica moderada. }\end{array}$ & $\begin{array}{l}\text { Relato de caso. } \\
\text { As sessões de hidroterapia foram } \\
\text { realizadas três vezes por semana } \\
\text { com duração de } 45 \text { minutos, durante } \\
20 \text { sessões. Avaliação do tônus } \\
\text { muscular. }\end{array}$ & $\begin{array}{l}\text { Apresentou redução na espasticidade, } \\
\text { proporcionou de uma forma geral uma } \\
\text { melhora no tônus muscular e na } \\
\text { qualidade de vida. }\end{array}$ \\
\hline
\end{tabular}

\footnotetext{
REAS/EJCH | Vol.Sup.30 | e728 | DOI: https://doi.org/10.25248/reas.e728.2019 Página 4 de 8
} 


\begin{tabular}{|c|c|c|c|c|c|}
\hline $\begin{array}{c}\text { BRANCHER } \\
\text { EC et al., } 2014 .\end{array}$ & $\begin{array}{l}\text { Método Halliwick: uma } \\
\text { proposta fisioterapêutica na } \\
\text { malformação congênita } \\
\text { induzida por isotretinoína }\end{array}$ & $\begin{array}{l}\text { Google } \\
\text { Acadêmico/Fisioterapia } \\
\text { Brasil }\end{array}$ & $\begin{array}{l}\text { Investigar os efeitos } \\
\text { do método Halliwick } \\
\text { sobre o } \\
\text { desenvolvimento } \\
\text { motor do recém- } \\
\text { nascido com } \\
\text { malformação } \\
\text { congênita decorrente } \\
\text { do uso de } \\
\text { isotretinoína. }\end{array}$ & $\begin{array}{l}\text { Relato de caso. Estudo exploratório } \\
\text { e quantitativo, com bebê de } 4 \text { anos } \\
\text { de idade com idade motora de } 6 \\
\text { meses. Foram realizadas } 30 \\
\text { sessões, divididas } 5 \text { sessões por } \\
\text { semana por } 40 \text { minutos cada, } \\
\text { avaliação da função motora grossa e } \\
\text { tônus muscular. }\end{array}$ & $\begin{array}{l}\text { Foi possível observar que o tratamento } \\
\text { mostrou benefícios em diversos } \\
\text { aspectos do } \\
\text { desenvolvimento motor do paciente, } \\
\text { sugerindo um efeito } \\
\text { positivo do método aplicado }\end{array}$ \\
\hline $\begin{array}{l}\text { RIBEIRO LF et } \\
\text { al., } 2015 .\end{array}$ & $\begin{array}{l}\text { A utilização da terapia } \\
\text { aquática como método de } \\
\text { redução da dor em UTI } \\
\text { Neonatal (Relato de caso) }\end{array}$ & $\begin{array}{l}\text { Google Acadêmico/ } \\
\text { VIII World Congress on } \\
\text { Communication and Arts }\end{array}$ & $\begin{array}{l}\begin{array}{l}\text { Uso da } \\
\text { aquática terapia } \\
\text { como }\end{array} \\
\text { método de redução } \\
\text { da dor em bebê } \\
\text { hospitalizado na } \\
\text { Unidade de Terapia } \\
\text { Intensiva Neonatal. }\end{array}$ & $\begin{array}{l}\text { Relato de caso, bebê de } 3 \text { meses. } \\
\text { Foram realizadas } 5 \text { sessões de } \\
\text { terapia aquática em balde do tipo } \\
\text { ofurô, iniciado as sessões } 1 \text { dia } \\
\text { antes do procedimento de } \\
\text { traqueostomia, sendo uma sessão } \\
\text { ao dia à temperatura de } 36^{\circ} \mathrm{C} \\
\text { durante } 10 \text { minutos. } \\
\text { Avaliação da dor por escala NIPS. }\end{array}$ & $\begin{array}{l}\text { No caso relatado após cada sessão de } \\
\text { terapia aquática, observou-se melhora } \\
\text { na frequência cardíaca, frequência } \\
\text { respiratória e saturação periférica de } \\
\text { oxigênio. A aplicação da escala da dor } \\
\text { NIPS } \\
\text { demonstrou um escore médio de } 4 \\
\text { (quatro) antes da terapia, } \\
\text { sendo reduzido para escore médio de } 1 \\
\text { (um) após a terapia } \\
\text { aquática. A terapia aquática é um } \\
\text { recurso que pode ser empregado no } \\
\text { tratamento da dor dos RNs, pois } \\
\text { proporciona estabilidade nos sinais } \\
\text { vitais desses indivíduos, além de bem- } \\
\text { estar e relaxamento. }\end{array}$ \\
\hline $\begin{array}{c}\text { BARBOSA } \\
\text { LPC, } \\
\text { CARNEIRO } \\
\text { EM, WEFFORT } \\
\text { V., } 2015 .\end{array}$ & $\begin{array}{l}\text { Impacto da hidroterapia em } \\
\text { recém-nascidos } \\
\text { hospitalizados }\end{array}$ & $\begin{array}{l}\text { Google Acadêmico/ } \\
\text { Revista Fisioterapia } \\
\text { Brasil }\end{array}$ & $\begin{array}{l}\text { Avaliar impacto da } \\
\text { hidroterapia em } 10 \\
\text { recém-nascidos } \\
\text { hospitalizados }\end{array}$ & $\begin{array}{l}\text { Estudo quase experimental, com } 10 \\
\text { RNs. Os RNs foram avaliados dois } \\
\text { dias consecutivos no primeiro dia, } \\
\text { realizada a verificação } \\
\text { da FC, FR, SAT e escala de dor } \\
\text { NIPS as } 7 \text { h30min e as } 8 \mathrm{~h}, 0 \\
\text { RN não foi submetido a nenhum } \\
\text { procedimento. No segundo } \\
\text { dia, o mesmo RN foi submetido a } \\
\text { verificação dos parâmetros }\end{array}$ & $\begin{array}{l}\text { Após a realização da hidroterapia } \\
\text { houve diminuição } \\
\text { das médias da FR e da FC e aumento } \\
\text { das médias da SAT O2. A avaliação da } \\
\text { dor } \\
\text { (NIPS) mostrou redução significativa } \\
\text { após a intervenção. }\end{array}$ \\
\hline
\end{tabular}

REAS/EJCH | Vol.Sup.30 | e728 | DOI: https://doi.org/10.25248/reas.e728.2019 Página 5 de 8 


\begin{tabular}{|c|c|c|c|c|c|}
\hline & & & & $\begin{array}{l}\text { fisiológicos e escala de dor NIPS nos } \\
\text { horários descritos e, após } \\
\text { a primeira coleta das respectivas } \\
\text { variáveis, foi submetido a } \\
\text { hidroterapia por um período de dez } \\
\text { minutos. Avaliação da FR, FC, } \\
\text { SpO2, dor. }\end{array}$ & \\
\hline $\begin{array}{l}\text { TOBINAGA } \\
\text { WCO et al., } \\
2016 .\end{array}$ & $\begin{array}{l}\text { Short-Term effects of } \\
\text { hydrokinesiotherapy in } \\
\text { hospitalized preterm } \\
\text { newborns }\end{array}$ & $\begin{array}{l}\text { PubMed/ Rehabilitation } \\
\text { Research and Practice }\end{array}$ & $\begin{array}{l}\text { Avaliar os efeitos a } \\
\text { curto prazo da } \\
\text { hidrocinesioterapia } \\
\text { na redução do } \\
\text { estresse no recém- } \\
\text { nascido pré-termo. }\end{array}$ & $\begin{array}{l}\text { Ensaio clínico não randomizado, } \\
\text { com } 15 \text { prematuros. Sinais vitais, dor } \\
\text { e saliva avaliados antes e após a } \\
\text { terapia com duração de } 10 \text { minutos } \\
\text { durante } 15 \text { dias }\end{array}$ & $\begin{array}{l}\text { Houve diminuição dos níveis de cortisol } \\
\text { após a terapia, diminuição significativa } \\
\text { na frequência cardíaca, e na frequencia } \\
\text { respiratória, aumento da SAT de O2, } \\
\text { pouca diferença na escala da dor NIPS. } \\
\text { A hidrocinesioterapia pode ser uma } \\
\text { alternativa terapêutica em } \\
\text { recém-nascidos pré-termo, sendo } \\
\text { tecnicamente fácil de administrar e } \\
\text { de baixo custo. }\end{array}$ \\
\hline $\begin{array}{l}\text { VALIZADEH C } \\
\text { et al., } 2017 .\end{array}$ & $\begin{array}{l}\text { Effect of early physical } \\
\text { activity programs on motor } \\
\text { performancer and } \\
\text { neuromuscular development } \\
\text { in infants born preterm: a } \\
\text { randomized clinical trial. }\end{array}$ & $\begin{array}{l}\text { PubMed/ Journal of } \\
\text { Caring Sciences }\end{array}$ & $\begin{array}{l}\text { Avaliar o efeito de } \\
\text { programas de } \\
\text { atividade física } \\
\text { dentro e fora da água } \\
\text { sobre o desempenho } \\
\text { motor e } \\
\text { desenvolvimento } \\
\text { neuromuscular em } \\
\text { bebês prematuros. }\end{array}$ & $\begin{array}{l}\text { Ensaio clínico randomizado, } 76 \\
\text { prematuros foram distribuídos } \\
\text { aleatoriamente em quatro grupos. } \\
\text { G1: movimentação passiva com } \\
\text { base no protocolo de Moyer-Mileur. } \\
\text { G2: hidroterapia (exercícios para os } \\
\text { ombros e área pélvica na água todos } \\
\text { os dias. } \\
\text { G3: combinação de programas de } \\
\text { atividade física dentro e fora da água } \\
\text { em dias alternados. G4: contenção } \\
\text { em posição fetal. Durante duas } \\
\text { semanas }\end{array}$ & $\begin{array}{l}\text { Houve pouca diferença entre os grupos } \\
\text { no desempenho neuromuscular. } \\
\text { Apenas o recuo da perna mostrou-se } \\
\text { significativamente diferente entre os } \\
\text { grupos de atividade física e } \\
\text { hidroterapia, mostrando melhor } \\
\text { resultado com a hidroterapia. }\end{array}$ \\
\hline
\end{tabular}

NFCS= Neonatal Facial Coding; NIPS= Neonatal Infant Pain Scale; RN= Recém-nascido, FR= Frequência Respiratória, FC= Frequência Cardíaca, SAT O2= Saturação Periférica de Oxigênio, UTI Neonatal= Unidade de Terapia Intensiva Neonatal, AIMS= Escala Motora Infantil.

Fonte: elaborado pelas autoras, 2019. 


\section{DISCUSSÃO}

Dos oito artigos analisados nesta revisão integrativa observa-se que três são do tipo relato de caso, dois são do tipo experimental, dois são estudos não randomizados e um ensaio clínico randomizado. A maioria dos artigos se propõe a avaliar os efeitos da fisioterapia aquática em relação a dor, estresse e sono em recémnascidos, exceto dois estudos que avaliam o desempenho motor dos bebês. A terapia aquática tem mostrado redução da frequência cardíaca e respiratória, melhora no sono e diminuição da dor, aumento da saturação periférica de oxigênio, assim como bons resultados no desenvolvimento motor de bebês, melhorando tônus muscular e qualidade de vida (VIGNOCHI C, et al., 2010; RIBEIRO LF, et al.,2015; BARBOSA LPC, et al., 2015; TOBINAGA WCO, et al., 2016; SILVA JO, et al., 2009; MELO FR, et al., 2012).

O uso da fisioterapia aquática é tolerado em bebês, pois é uma técnica segura e teve um efeito positivo na redução da dor, melhora da qualidade do sono e aumento de peso. Durante o procedimento, os recémnascidos demonstraram mais tranquilidade, diminuíram o choro, olhos abertos e movimentos corporais mais suaves (VALIZADEH L, et al., 2017).

Os efeitos fisiológicos da água produzem diminuição da dor e relaxamento muscular, devido ao aumento da circulação sanguínea e diminuição dos terminais nervosos (VIGNOCHI C, et al., 2010).

Para Tobinaga WCO, et al. (2016); Barbosa LPC, et al. (2015); Ribeiro LF, et al. (2015); Vignochi C, et al. (2016) o método da intervenção não ultrapassou o tempo de 10 minutos de terapia, o que sugere ser um período suficiente e tolerável para RNs, a técnica entre os estudos foi parecida, imersão até os ombros, movimentos lentos proporcionando conforto e segurança.

Outros assuntos foram abordados com aplicação da fisioterapia aquática: na estimulação do desenvolvimento motor de bebês 18 meses; com malformação congênita e também reabilitação com efeito da atividade física dentro e fora da água sobre o desempenho motor e desenvolvimento. Esses estudos mostraram que a terapia aquática melhora a espasticidade e tônus muscular de crianças com malformação congênita, a estimulação na água facilita o desenvolvimento motor das crianças de 18 meses, assim como melhora a qualidade de vida (SILVA JO, et al., 2009; BRANCHER EC, et al., 2014; VALIZADEH L, et al., 2016).

Métodos diferentes de terapia com tempo maior e priorizando exercícios, estimulação e atividades aquáticas também são bem aceitos. Nesse sentido a fisioterapia aquática foi usada não somente para RNs, mas para crianças também mostrando ser benéfica para o desenvolvimento motor e melhor qualidade de vida (VALIZADEH L, et al., 2017; BRANCHER EC, et al., 2014; MELO FR, et al., 2012; SILVA JO, et al., 2009).

Vignochi C, et al. (2010); Barbosa LPC, et al. (2015); Tobinaga WCO, et al. (2016), mostraram resultados semelhantes nos efeitos da fisioterapia aquática em recém-nascidos prematuros hospitalizados, após a terapia os bebês apresentaram queda da FR, FC, aumento da SpO2 e diminuição da dor.

A reabilitação aquática é proposta para vários tipos de tratamentos, incluindo também tratamento de lesões cerebrais e seus problemas associados, como fraquezas musculares, déficit de coordenação motora e equilíbrio (MELO FR, et al., 2012).

A fisioterapia aquática, tem se tornado uma terapêutica aceita por muitas pessoas, pois proporciona manobras mais leves dentro da água, onde muitas vezes seriam difíceis de realizá-las fora da água (JACQUES KC, et al., 2010).

O banho de imersão como fisioterapia aquática, é indicado, pois seus efeitos produzem alívio da dor e relaxamento muscular. Isso ocorre devido a diminuição da sensibilidade das terminações nervosas, também com o aumento da circulação sanguínea, aumentando o estímulo sanguíneo aos músculos, inclusive musculatura respiratória, e assim ocorre o aumento da capacidade vital, promovendo bem-estar e conforto.

Também foi observado neonatos que tiveram dificuldades durante o período de amamentação, uma melhoria visível da sucção após uso da técnica de banho de imersão ou ofurô, uma vez que ficam mais relaxados depois de terminar o banho (PERINE C, et al., 2014; ATAÍDE VP, et al., 2016). 


\section{CONSIDERAÇÕES FINAIS}

Os resultados demonstraram que a fisioterapia aquática constitui um recurso seguro para sua aplicação em recém-nascidos e crianças em diversas temáticas como: dor, desenvolvimento neuromotor e bem-estar. A técnica deve ser feita com indicações precisas e cautela na sua utilização, sendo sempre realizada por um profissional capacitado, também é necessário ter cuidado com a temperatura da água e assepsia da banheira e controle dos sinais vitais. No entanto, foi possível evidenciar a eficácia da fisioterapia aquática em recémnascidos ou crianças. Dessa forma, pela presente revisão são necessários estudos futuros para que novas referências teóricas colaborem para uma conduta clínica de melhor qualidade da abordagem terapêutica na água nessa população.

\section{REFERÊNCIAS}

1. ATAÍDE VP, BARBOSA JSV, CARVALHO MGS, NEVES SMSG, SANCHEZ FF, GONÇALVEZ RL. Ofurô em recémnascidos pré-termo de baixo peso: relato de experiência. Assobrafir Ciência. 2016 Ago;7(2):13-22.

2. BARBOSA LPC, CARNEIRO EM, WEFFORT V. Impacto da Hidroterapia em recém-nascidos hospitalizados. Fisioter Bras. 2015 Nov; 16(3): 207-11.

3. BARBOSA, LPC. Estimate of hydrotherapy benefits in hospitalizeds newborns. 2012. 67 f. Dissertação (Mestrado em Atenção à Saúde das Populações) - Universidade Federal do Triângulo Mineiro, Uberaba, 2012.

4. BRANCHER EC, RODRIGUES RP, FRANCO PS, ROCHA ES, MACHADO A, CHIQUETTI EMS, et al. Método Halliwick: uma proposta fisioterapêutica na malformação congênita induzida por isotretinoína. Fisioter Bras. 2014 Ago; 15(14): 283-87.

5. BRICKHOUSE B, ISAACS C, BATTEN M, PRICE A. Strategies for providing low-cost water immersion therapy with limeted resources. Nurs Womens Health. 2016 Jan; 19 (6): 526-32.

6. CARVALHO DR, BRAGA EM, LOCHINI AF, PROBST VS, PITTA F, FELCAR JM. Avaliação da capacidade funcional de exercícios no ambiente aquático. Fisioter e Pesq. 2015 Nov; 22 (4): 355-62.

7. JACQUES KC, DRUMOND NR, ANDRADE SAF, JÚNIOR JPC, TOFFOL WC. Eficácia da hidroterapia em crianças com encefalopatia crônica não progessiva da infância: revisão sistemática. Fisioter Mov. 2010 Mar; 23(1):53-61.

8. MELO FR, ALVES DAG, LEITE JMS. Benefícios da hidroterapia para espasticidade em uma criança com hidrocefalia. Rev Neurocienc. 2012 Abr; 20(3): 415-21.

9. PASCUAL CT. Valor de La hidroterapia em La cultura fenícia. Ver Um Med. 2014 Sep; 14(3):751-6.

10. PERINI C, SEIXAS MC, CATÃO ACSM, SILVA GD, ALMEIDA VS, MATOS PBC. Banho de ofurô em recém-nascidos no alojamento conjunto: um relato de experiência. J. Res Fundam Care Online. 2014 Jun;6(2): 785-92.

11. RIBEIRO LF, XAVIER GN, KAIRALA ALR, OLIVEIRA MS. A utilização da terapia aquática como método de redução da dor em UTI Neonatal (Relato de caso). VIII World Congress on Communication and Arts. 2015 Abril; 19 (22): 313-16.

12. SILVA JO, MARTINS JC, MORAIS RLS, GOMES WF. Influência da estimulação aquática no desenvolvimento de crianças de 0 a 18 meses: um estudo piloto. Fisioter e Pesq. 2009 Dez; 16(4): 335-40.

13. TOBINAGA WCO, MARINHO CL, ABELENDA VLB, SÁ PM, LOPES AJ. Short-term effects of hidrokinesiotherapy in hospitalized preterm newborns. Reabilitation on Research and Pratice. 2016 Ago; 2016:1-8.

14. VALIZADEH L, SANAEEFAR M, HOSSEINI MB, JAFARABADI MA, SHAMILI A. Effect of early physical activity programs on motor performance and neuromuscular development in infants born preterm: a randomized clinical trial. J. Caring Sci. 2017 Mar; 6 (6): 67-79.

15. VIGNOCHI C, TEIXEIRA PP, NADER SS. Efeito da fisioterapia aquática na dor e no estado de sono e vigília de recém-nascidos pré- termo estáveis internados em unidade de terapia in tensiva neonatal. Rev Bras Fisioter. 2010 maio/Jun; 14(3): 214-20. 